Proceedings of the International Symposium on Physics of Materials (ISPMA 14), September 10-15, 2017, Prague

\title{
Characterization of Active Deformation Mechanisms in Mg Alloys with LPSO Phase
}

\author{
K. Horváth ${ }^{a, b, *}$, D. Drozdenko ${ }^{a}$, K. MÁthis ${ }^{a}$, G. Garcés ${ }^{c}$ And P. Dobroñ ${ }^{a}$ \\ ${ }^{a}$ Charles University, Faculty of Mathematics and Physics, Department of Physics of Materials, \\ Ke Karlovu 5, 12116 Prague, Czech Republic \\ ${ }^{b}$ Nuclear Physics Institute of the CAS, 25068 Rež, Czech Republic \\ ${ }^{c}$ CENIM-CSIC, Department of Physical Metallurgy, Avenida Gregorio del Amo 8, E-28040 Madrid, Spain \\ The WZ21 and the WZ72 magnesium alloys containing long period stacking ordered (LPSO) phase extruded \\ with an extrusion ratio of $4: 1$ at $350^{\circ} \mathrm{C}$. The microstructure was analyzed by scanning electron microscopy using \\ electron backscattered diffraction. Both alloys exhibit bimodal microstructure of the $\mathrm{Mg}$ matrix and elongated \\ LPSO phase along the extrusion direction. Using kernel average misorientation (KAM) maps it was shown that \\ the dynamic recrystallization starts at the grain boundaries of the initial coarse grains. Both alloys exhibit fiber \\ basal texture. The maxima of the basal planes are reduced by increasing content of alloying elements. Uniaxial \\ compression tests along extrusion direction with concurrent acoustic emission measurements were performed to \\ reveal mechanical properties and active deformation mechanisms of the studied alloys. The acoustic emission source \\ mechanisms were identified by statistical analysis of the raw acoustic emission signal.
}

DOI: 10.12693/APhysPolA.134.815

PACS/topics: 62.20.F-, 62.20.M-, 81.05.Bx, 43.40.Le

\section{Introduction}

Nowadays, magnesium $(\mathrm{Mg})$ alloys with long-period stacking-ordered (LPSO) phase received high scientific interest due to their improved mechanical properties compared to commercial $\mathrm{Mg}$ alloys. After simple hot extrusion process, a $\mathrm{Mg}-\mathrm{Y}-\mathrm{Zn}$ ternary alloy can exhibit yield stress higher than $300 \mathrm{MPa}$ and reasonable ductility between 5 and $10 \%[1-3]$.

During extrusion, a significant texture with basal planes oriented parallel to the extrusion direction (ED) is formed. This kind of texture promotes nucleation of (10-12) extension twinning when compression loading is applied along ED [4-6]. The plasticity of the Mg alloys is mainly controlled by basal and prismatic $\langle a\rangle$ slip [7]. The activation of a $\langle c+a\rangle$ slip is rather difficult owing to high critical resolved shear stress (CRSS), and therefore deformation twinning is needed to accommodate the deformation out of the basal plane. On the other hand, the presence of the LPSO phase, which is formed by chemically ordered stacking faults, may delay or inhibit twin nucleation in the $\mathrm{Mg}$ matrix $[8,9]$.

The deformation mechanisms of the LPSO phase were also investigated. It is believed that the LPSO phase deforms mainly by $(0001)\langle 11 \overline{2} 0\rangle$ basal slip [2]. It was showed that Young's modulus of the LPSO phase $(\approx 67 \mathrm{GPa})$ is higher than that of the Mg matrix $(\approx 40 \mathrm{GPa})[10,11]$. Consequently, the LPSO phase strengthens the material. According to Hagihara [12], the strengthening mechanisms are similar

*corresponding author; e-mail: horvathk@karlov.mff.cuni.cz to the short-fiber reinforced composites, where load transfer from the matrix to the stiffer fibers takes place.

The deformation behavior of $\mathrm{Mg}$ alloys with the LPSO phase is not completely understood, yet. Therefore, we applied acoustic emission (AE) measurements in order to reveal active deformation mechanisms during compression loading. Despite the different character of the AE signals from individual sources: twinning produces a burst signal, whereas the dislocations slip a continuous one; it is not an easy task to separate them. Several statistical algorithms were developed for this purpose [13-15]. In this work, we used an adaptive sequential $k$-means (ASK) procedure developed by Pomponi and Vinogradov [15]. It determines the dominant deformation mechanism in a given time period by processing the raw (not filtered) AE signal.

The initial microstructure was studied by using scanning electron microscopy (SEM). Inverse pole figure (IPF) maps and kernel average misorientation (KAM) maps were calculated using electron backscatter diffraction (EBSD) measurements. The KAM values are good indicators of localized strain in materials and are useful for characterization of the initial state of the materials [16].

\section{Experimental methods}

The WZ21 $(\mathrm{Mg}+1.8 \mathrm{wt} \% \mathrm{Y}+0.7 \mathrm{wt} \% \mathrm{Zn}$, $\mathrm{Mg}_{97} \mathrm{Y}_{0.5} \mathrm{Zn}_{0.25}$ in at.\%) and the WZ72 $(\mathrm{Mg}+6.9 \mathrm{wt} \% \mathrm{Y}$ +2.5 wt\% $\mathrm{Zn}, \mathrm{Mg}_{97} \mathrm{Y}_{2} \mathrm{Zn}_{1}$ ) magnesium alloys having an addition of 0.5 wt\% of $\mathrm{CaO}$ were cast in KITECH (Korea). The cast billets were extruded with an extrusion speed of $0.5 \mathrm{~mm} / \mathrm{s}$ and an extrusion ratio of $4: 1$ at $350^{\circ} \mathrm{C}$ in Centro Nacional de Investigaciones Metalúrgicas (CENIM) Madrid. 
The deformation tests were carried out using a universal testing machine INSTRON ${ }^{\circledR} 5882$ at room temperature (RT) with a constant strain rate of $10^{-3} \mathrm{~s}^{-1}$. The samples with a length of $15 \mathrm{~mm}$ and a diameter of $10 \mathrm{~mm}$ were compressed along ED.

The AE activity during deformation tests was monitored by a computer-controlled MICRO-II system developed by Physical Acoustics Corporation (PAC), which allows a continuous storage of AE signals with a sampling frequency of $2 \mathrm{MHz}$. A piezoelectric MIDI-410-61 sensor (ZD RPETY-DAKEL) with a diameter of $6 \mathrm{~mm}$ was used. The AE sensor was attached to the sample with a help of a clamp. High signal/noise ratio was ensured by using a $60 \mathrm{~dB}$ preamplifier.

For microscopic observations, the samples were ground on $\mathrm{SiC}$ papers and subsequently polished by diamond pastes down to $0.25 \mu \mathrm{m}$ particle size. Finally, the specimens' surface was polished by argon ions (Gatan PIPS ${ }^{\mathrm{TM}}$ ) which enable EBSD measurements. The EBSD map- ping was provided at FEI Quanta 200 FX scanning electron microscope equipped with EDAX EBSD camera and OIM software was utilized for EBSD observations.

\section{Results}

The EBSD IPF maps of the extruded WZ21 and WZ72 alloys are presented in Fig. 1a and d, respectively. Non-indexed areas correspond to confidence in$\operatorname{dex}(\mathrm{CI})<0.1$ which indicates on multiple diffractions and thus an occurrence of the LPSO phase. The microstructure of both alloys is rather complex. It contains the LPSO phase elongated along ED, Mg-matrix having a bimodal microstructure with coarse grains elongated along ED and small dynamically recrystallized (DRX) grains. The volume fraction of the LPSO phase and that of the DRX grains are higher in the WZ72 alloy than in the WZ21 alloy. The grain size of the DRX grains is $(1.23 \pm 0.06) \mu \mathrm{m}$ for the WZ21 alloy and $(1.08 \pm 0.04) \mu \mathrm{m}$ for the WZ72 alloy.
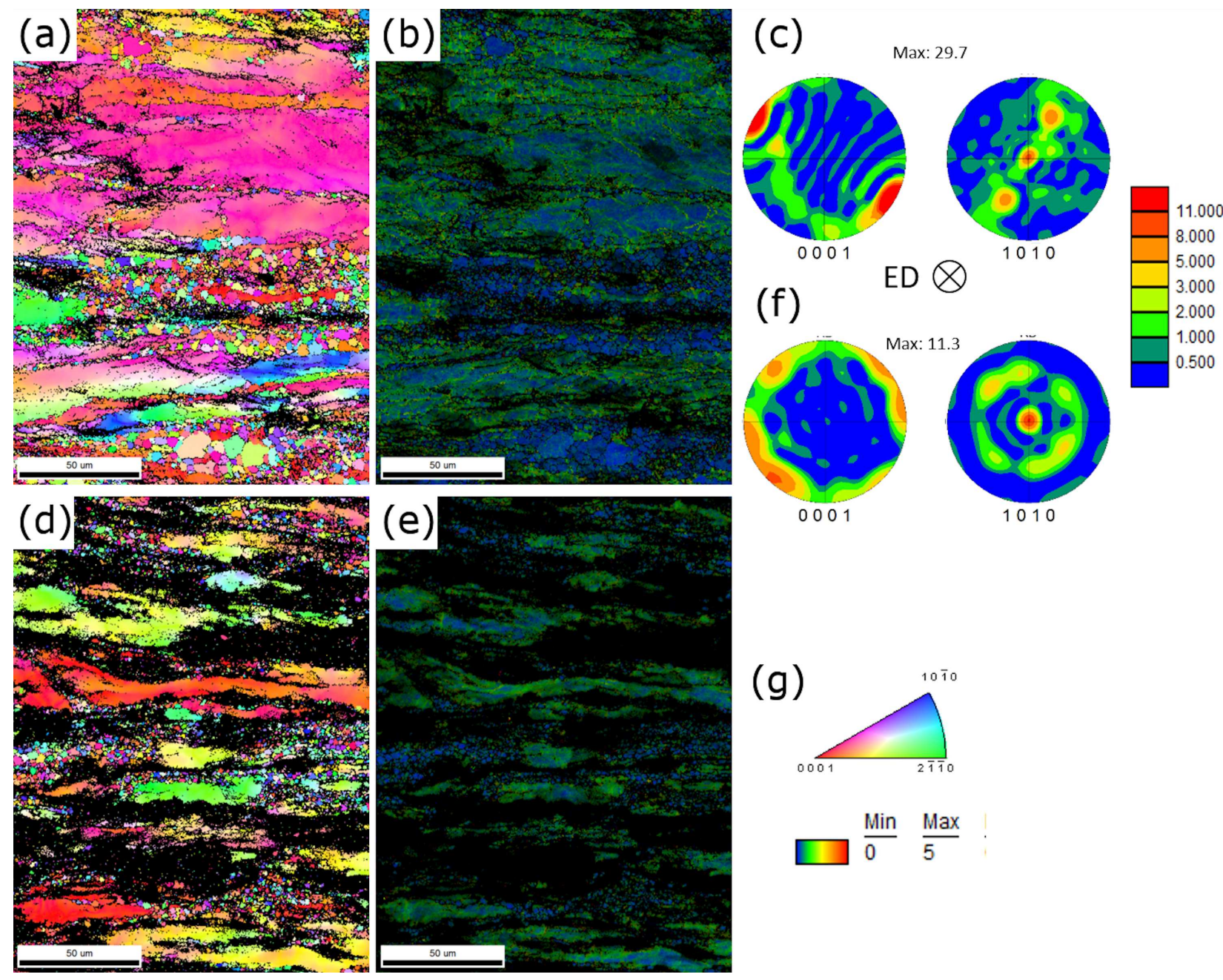

Fig. 1. EBSD inverse pole figure map $(\mathrm{ED} \rightarrow)$ of the longitudinal section, the KAM map and the texture of the WZ21 alloy are represented in (a)-(c), and that of the WZ72 alloy in (d)-(f), respectively. The scale of the texture intensities is carried out as the multiples of a random density (m.r.d.) from 0-11.000 with maximum intensity of 29.7 m.r.d. for the WZ21 alloy and 11.3 m.r.d. for the WZ72 alloy. The orientation triangle for the EBSD maps and the scale bar for KAM maps are shown in $(\mathrm{g})$. The maximum of the KAM scale is $5^{\circ}$ misorientation. 
In Fig. $1 \mathrm{~b}$ and e the KAM maps of the WZ21 and the WZ72 alloys are shown. The KAM value is very heterogeneous in the coarse grains for both alloys. In the WZ21 alloy with wide coarse grains, KAM values increase from the center of the grains to the edge. The DRX grains are more homogeneous compared to the coarse ones.

The (0001) and (1010) pole figures calculated from the IPF maps are shown in Fig. 1c and $\mathrm{f}$ for the WZ21 and the WZ72 alloys, correspondingly. In the WZ21 alloy, the basal planes form two distinct maxima in the (0001) pole figure with higher intensity than that in the WZ72 alloy. In the (1010) pole figure, three main maxima can be seen; one of them is in the center of the pole figure and two others symmetrically tilted by $30^{\circ}$ from the center. The WZ72 alloy also has a basal texture with basal planes symmetrically distributed around ED in the (0001) pole figure.

The time dependence of the AE signal and the compression stress are plotted in Fig. 2a for the WZ21 alloy and in b for the WZ72 alloy deformed along ED. The deformation curve of the WZ21 alloy has an S-shape, typically observed during compression of extruded Mg alloys. The stress plateau after the yield point is followed by rapid strain hardening. This effect is less pronounced for the WZ72 alloy and the inflection point is shifted towards higher stresses. The yield stress and ultimate compression stress are higher for the WZ72 alloy than for the WZ21 alloy.
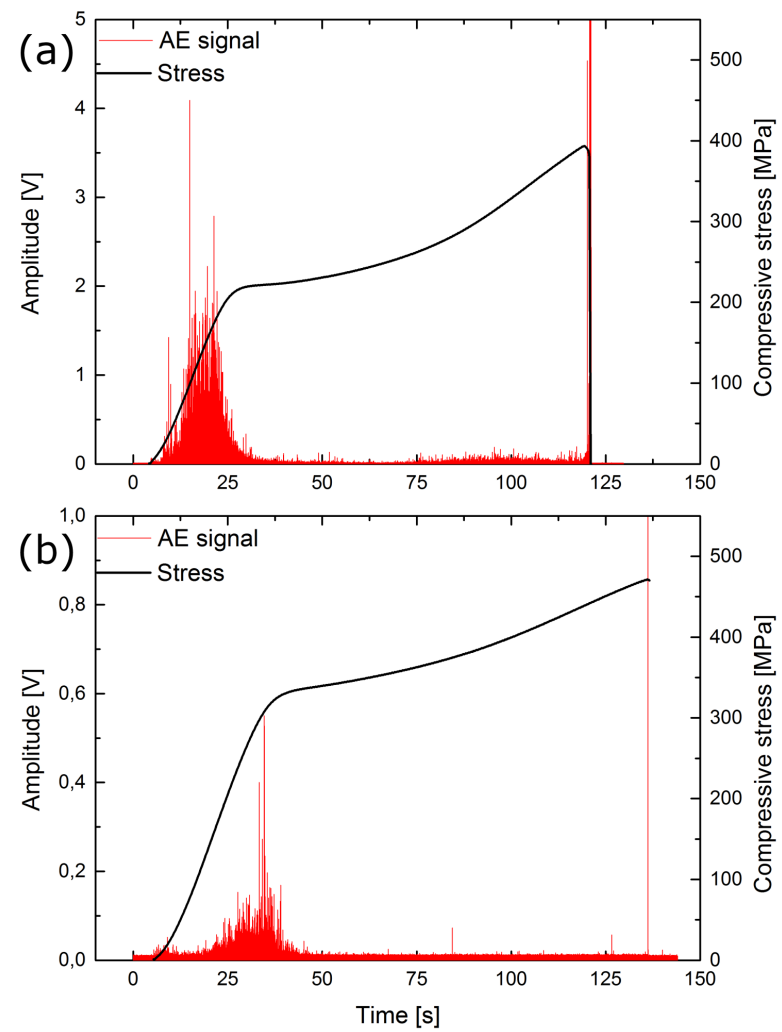

Fig. 2. Compression test of (a) the WZ21 alloy and (b) the WZ72 alloys deformed along ED with a concurrent AE measurement.

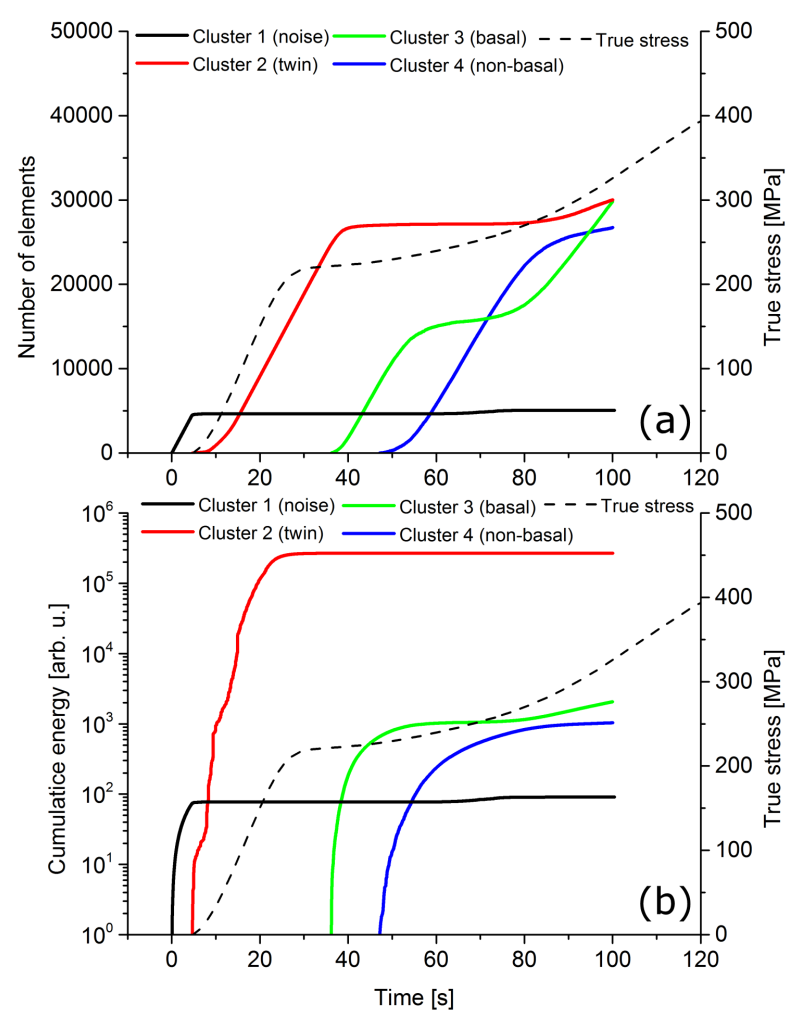

Fig. 3. The number of elements (i.e. the cumulative number of time frames assigned to each cluster) (a) and the cumulative energy (b) vs. time for the WZ21 alloy compressed along ED.

Stronger AE signal (higher amplitudes) are measured during deformation of the WZ21 alloy (please note the different scale in Fig. 2). The maximum of the AE response is measured slightly before the yield point and its intensity rapidly decreases afterward in both alloys. During plastic deformation, only a few low amplitude signals are detected.

Figure 3 shows the time evolution of the cumulative number of elements (a) and the cumulative energy (b) of different clusters for the compression loading of the WZ21 alloy. Figure 4 shows the same for the WZ72 alloy. The significant difference between the time evolution of different clusters helps to link deformation mechanisms to them. Cluster 1 (noise) naturally occurs first, already before the start of the experiment. When deformation starts other clusters are activated. The number of elements in cluster 1 (noise) starts to increase again when the $\mathrm{AE}$ amplitudes are very low (see Fig. 2).

This underlines that the signal of the respective mechanism dominates the assignment to a specific cluster. It does not mean that the mechanisms that do not dominate the AE signals are not active. They just do not dominate the signal at that moment.

In the WZ21 alloy (Fig. 3), at the onset of straining an increase of the number of elements in cluster 2 (twinning) is found. After the yield point, during the stress plateau, a distinct increase in the number of cluster 3 elements (basal slip) takes place. With proceeding plastic 

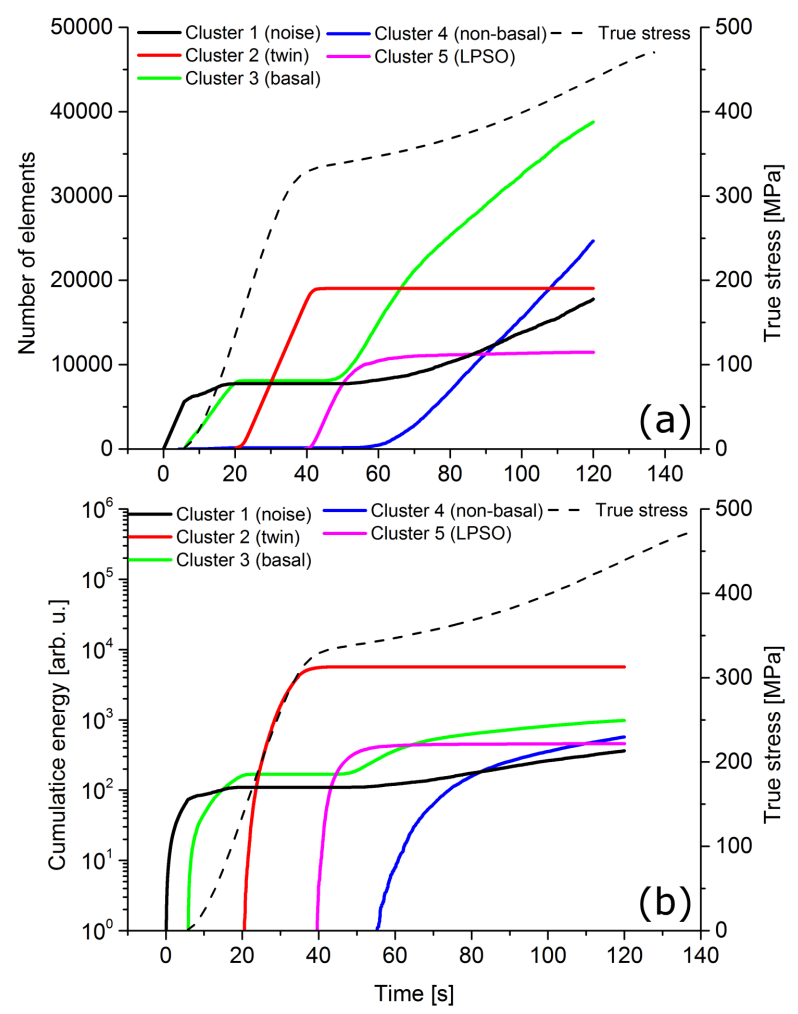

Fig. 4. As in Fig. 3, but for the WZ72 alloy.

deformation, the number of elements in cluster 4 (nonbasal slip) starts to increase. After the inflection point, a number of elements of all clusters start to increase again.

During the compression of the WZ72 alloys (Fig. 4), first, the number of elements in cluster 3 (basal slip) start to grow. Before the yield point, cluster 2 (twinning) takes over the dominance of the AE signal till its offset at the yield point. At this point, the number of elements in cluster 3 (basal slip) and cluster 5 (LPSO) starts to increase. At the later stage of deformation, the number of elements of cluster 3 (basal slip) and cluster 4 (non-basal slip) increases until the end of the deformation simultaneously.

The development of the cumulative energies (Figs. $3 \mathrm{~b}$ and $4 \mathrm{~b}$ ) of different clusters is qualitatively the same as the case of the number of elements. However, it can help to reveal which deformation mechanism gives a higher impact to the deformation than others.

\section{Discussion}

The bimodal microstructure observed in Fig. 1 is the result of the applied extrusion process. It was reported in [4] that the low extrusion speed and/or low extrusion ratio could lead to the formation of such microstructures. It was suggested in [17] that during extrusion DRX grains firstly occur at the edge of the initial coarse grains and then develops towards the inner parts. This statement is in good agreement with our results and with the KAM maps in Fig. 1. The high KAM values in the initial coarse grains suggest that large plastic strain is stored in them before the start of the DRX process. DRX releases energies accumulated by plastic strain and refines the grain size [18]. The KAM values are significantly lower in the center of the large grains of the WZ21 alloy than at their boundaries. Therefore, the DRX process starts at the edge of the initial grains and high angular grain boundaries are formed. Some DRX grains can be found with high KAM values what indicates that these grains underwent further deformation. It can be summarized that with increasing content of alloying elements the volume fraction of the initial coarse grains is reduced at the expense of the DRX grains and the LPSO phase.

Both alloys exhibit a typical basal texture with basal planes oriented parallel to ED. It was shown in some studies $[1,8]$ that the main contributors to this texture are the initial coarse grains. Therefore, the reduction of their volume fraction results in texture weakening of the WZ72 alloy compared to the WZ21 alloy.

The S-shaped compression curve of the WZ21 alloy is a typical sign of twinning activity. It was reported in $[8,9]$ that the presence of the LPSO phase can inhibit or delay twin formation in the $\mathrm{Mg}$ matrix. It is consistent with the shape of the deformation curve for the WZ72 alloy. It is also known that the high twinning activity results in lower yield stress [19]. As it was mentioned, the LPSO phase strengthens the material. These statements are consistent with the higher yield and ultimate compression stress for the WZ72 alloy than for the WZ21 alloy.

It is well documented that twin activation is an excellent source of AE, generating high amplitude AE signals [20, 21]. From the raw AE signal, presented in Fig. 2, we can assume that twinning controls the yield in the WZ21 alloy. Contrary to twin nucleation their growth cannot produce detectable AE signal [22, 23]. Moreover, newly formed twins are obstacles for moving dislocations and thus the reduction of their mean free path reduces the AE signal [5]. These effects result in the low AE response above the yield point.

The AE signal detected for the WZ72 alloy is significantly lower compared to the WZ21 alloy. The influence of the grains size on AE response is well documented $[5,19]$. As the result of a high fraction of grain boundaries, which are obstacles for dislocation motion, the AE response decreases with decreasing average grain size. The lower fraction of coarse grains in the WZ72 alloy compared to the WZ21 alloy reduces the amplitude of the AE signal.

The findings above are verified by the results of ASK analysis. The WZ21 alloy favors twin nucleation as the dominant mechanism at the yield point. From the high cumulative energy of twinning, it can be assumed that twinning takes place in the initial coarse grains, which are well oriented for activation of (1012) extension twinning. When twin nucleation is not the dominant mechanism anymore (after the yield), basal slip grows to some significance. It is well documented that extension twinning causes a crystal lattice re-orientation of $86.3^{\circ}$ around the $(10 \overline{1} 2)$ plane and plastic deformation is realized by a 
dislocation slip in the reoriented basal planes [24]. As the re-orientation of basal planes continues basal slip is not enough to accommodate the strain anymore and nonbasal slip is activated. The later stage of deformation is controlled by the simultaneous activity of the basal and non-basal slip systems.

The first dominant deformation mechanism in the WZ72 alloy is the basal slip. Similarly to the WZ21 alloy, the yield is controlled by twinning. Contrary, the number of twinning elements is significantly lower compared to the WZ21 alloy due to the lower fraction of coarse grains and the higher fraction of the LPSO phase than in the WZ21 alloy. The cumulative energy of twinning is also lower than that of the WZ21 alloy which is in good agreement with a smaller grain size of the coarse grains. After the yield point when twin nucleation is exhausted, a new cluster, linked to the deformation of the LPSO phase, starts to be dominant in the AE signal. It was shown in some papers that Young's modulus of the LPSO phase is higher than that of the $\mathrm{Mg}$ matrix $[10,11]$. The onset of cluster 5, around $330 \mathrm{MPa}$, could be linked to the start of the deformation of the LPSO phase. The main deformation mechanism of the LPSO phase is believed to be the formation of kink bands $[2,3]$. Besides, the formation of microcracks was reported in $\mathrm{Mg}_{85} \mathrm{Zn}_{6} \mathrm{Y}_{9}$ alloy already around the yield [25]. The relatively low number of elements of cluster 5 has rather high cumulative energy, which suggests that besides kinking a microcracking of the LPSO phase could take place. When cluster 5 is exhausted the basal slip starts to be dominant again and with further deformation non-basal slip is activated, too.

\section{Conclusions}

- The small extrusion ratio of $4: 1$ and a slow extrusion speed of $0.5 \mathrm{~mm} / \mathrm{s}$ lead to a formation of the bimodal microstructure of $\mathrm{Mg}$ matrix. The KAM maps proved that the dynamic recrystallization starts at the grain boundaries of the coarse grains.

- The increasing amount of alloying elements increases the volume fraction of the LPSO phase and results in improved mechanical properties.

- Twinning plays a minor role in yielding of alloy with the higher volume fraction of the LPSO phase. The plastic deformation of the studied alloys is controlled by simultaneously active basal and nonbasal slip.

\section{Acknowledgments}

The authors are grateful for support from the Czech Science Foundation under grant No. 1612075S; the Grant Agency of the Charles University under grant No. 1262217; the grant SVV-2017260442; the Operational Programme Research, Development and Education, The Ministry of Education, Youth and Sports (OP RDE, MEYS) under the grant
CZ.02.1.01/0.0/0.0/16 013/0001794 and the Spanish Ministry of Economy and Competitiveness under the grant number MAT2012-34135.

\section{References}

[1] M. Yamasaki, K. Hashimoto, K. Hagihara, Y. Kawamura, Acta Mater. 59, 3646 (2011).

[2] K. Hagihara, N. Yokotani, Y. Umakoshi, Intermetallics 18, 267 (2010).

[3] K. Hagihara, Y. Sugino, Y. Fukusumi, Y. Umakoshi, T. Nakano, Mater. Trans. 52, 1096 (2011).

[4] J. Bohlen, S. Yi, D. Letzig, K.U. Kainer, Mater. Sci. Eng. A 527, 7092 (2010).

[5] P. Dobroň, F. Chmelík, S. Yi, K. Parfenenko, D. Letzig, J. Bohlen, Scr. Mater. 65, 424 (2011).

[6] J. Victoria-Hernandez, S. Yi, D. Letzig, D. Hernandez-Silva, J. Bohlen, Acta Mater. 61, 2179 (2013).

[7] S.R. Agnew, D.W. Brown, C.N. Tomé, Acta Mater. 54, 4841 (2006).

[8] G. Garces, P. Perez, S. Cabeza, H.K. Lin, S. Kim, W. Gan, P. Adeva, Mater. Sci. Eng. A 647, 287 (2015).

[9] X.H. Shao, Z.Q. Yang, X.L. Ma, Acta Mater. 58, $4760(2010)$.

[10] Y. Chino, M. Mabuchi, S. Hagiwara, H. Iwasaki, A. Yamamoto, H. Tsubakino, Scr. Mater. 51, 711 (2004).

[11] M. Tane, Y. Nagai, H. Kimizuka, K. Hagihara, Y. Kawamura, Acta Mater. 61, 6338 (2013).

[12] K. Hagihara, A. Kinoshita, Y. Sugino, M. Yamasaki, Y. Kawamura, H.Y. Yasuda, Y. Umakoshi, Acta Mater. 58, 6282 (2010).

[13] A. Kontsos, T. Loutas, V. Kostopoulos, K. Hazeli, B. Anasori, M.W. Barsoum, Acta Mater. 59, 5716 (2011).

[14] Y.P. Li, M. Enoki, Mater. Sci. Eng. A 536, 8 (2012).

[15] E. Pomponi, A. Vinogradov, Mech. Syst. Sign. Process. 40, 791 (2013).

[16] D.P. Field, C.C. Merriman, N. Allain-Bonasso, F. Wagner, Model. Simul. Mater. Sci. Eng. 20, 024007 (2012).

[17] C. Xu, T. Nakata, X. Qiao, M. Zheng, K. Wu, S. Kamado, Sci. Rep. 7, 40846 (2017).

[18] L. Jiang, W. Huang, D. Zhang, F. Guo, H. Xue, J. Xu, F. Pan, J. Alloys Comp. 727, 205 (2017).

[19] P. Dobroň, F. Chmelík, K. Parfenenko, D. Letzig, J. Bohlen, Acta Phys. Pol. A 122, 593 (2012).

[20] C.R. Heiple, S.H. Carpenter, J. Acoust. Emiss. 6, 177 (1987).

[21] D. Drozdenko, J. Bohlen, F. Chmelík, P. Lukáč, P. Dobroň, Mater. Sci. Eng. A 650, 20 (2016).

[22] C.R. Heiple, S.H. Carpenter, J. Acoust. Emiss. 6, 215 (1987).

[23] J.P. Toronchuk, Mater. Eval. 35, 51 (1977).

[24] F.A. Mirza, D.L. Chen, D.J. Li, X.Q. Zeng, Mater. Sci. Eng. A 575, 65 (2013).

[25] Y. Muto, T. Shiraiwa, M. Enoki, Mater. Sci. Eng. A 689, 157 (2017). 